\title{
A numerical algorithm for efficiently obtaining a Feynman parameter representation of one-gluon loop QCD Feynman diagrams for a large number of external gluons
}

\author{
A. S. Kapoyannis*, A. I. Karanikas and C. N. Ktorides \\ University of Athens, Physics Department \\ Nuclear $\& 3$ Particle Physics Section \\ Panepistimiopolis, Ilisia GR 157-71, Athens, Greece
}

\begin{abstract}
A numerical program is presented which facilitates a computation pertaining to the full set of one-gluon loop diagrams (including ghost loop contributions), with M attached external gluon lines in all possible ways. The feasibility of such a task rests on a suitably defined master formula, which is expressed in terms of a set of Grassmann and a set of Feynman parameters. The program carries out the Grassmann integration and performs the Lorentz trace on the involved functions, expressing the result as a compact sum of parametric integrals. The computation is based on tracing the structure of the final result, thus avoiding all intermediate unnecessary calculations and directly writing the output. Similar terms entering the final result are grouped together. The running time of the program demonstrates its effectiveness, especially for large $\mathrm{M}$.
\end{abstract}

PACS: 02.70.Rw, 12.38.Bx

*Corresponding author. E-mail address: akapog@phys.uoa.gr (A. S. Kapoyannis) 


\section{PROGRAM SUMMARY}

Manuscript title: A numerical algorithm for efficient computations of one-gluon loop Feynman diagrams in QCD for a large number of external gluons

Authors: A. S. Kapoyannis, A. I. Karanikas and C. N. Ktorides

Program title: DILOG2

Programming language: FORTRAN 90

Computer(s) for which the program has been designed: Personal Computer

Operating system(s) for which the program has been designed: Windows 98, XP, LINUX

Number of processors used: one

Keywords: one-gluon loop, Feynman diagram, QCD diagram

PACS: 02.70.Rw, 12.38.Bx

CPC Library Classification: 11.5 Quantum Chromodynamics, Lattice Gauge Theory

External routines/libraries used: none

CPC Program Library subprograms used: none

Nature of problem: The computation of one gluon/ghost loop diagrams in QCD with many external gluon lines is a time consuming task, practically beyond reasonable reach of analytic procedures. We apply recently proposed master formulas towards the computation of such diagrams with an arbitrary number $(M)$ of external gluon lines, achieving a final result which reduces the problem to one involving integrals over the standard set, for given $M$, of Feynman parameters.

Solution method: The structure of the master expressions is analysed from a numerical computation point of view. Using the properties of Grassmann variables we identify all the different forms of terms that appear in the final result. Each form is called "structure". We calculate theoretically the number of terms belonging to every "structure". We carry out the calculation organising the whole procedure into separate calculations of the terms belonging to every "structure". Terms which do not contribute to the final result are thereby avoided. The final result, extending to large values of $M$, is also presented with terms belonging to the same "structure" grouped together.

Restrictions: $M$ is coded as a 2-digit integer. Overflow in the dimension of used array is expected to appear for $M \geq 20$ in a processor that uses 4 -bytes integers or for $M \geq 34$ in a 
processor with 8-bytes integers.

Running time: Depends on $M$, see enclosed figures. 


\section{LONG WRITE-UP}

\section{Introduction}

Quantum Chromodynamics (QCD) enjoys universal acceptance as the fundamental theory for the strong interaction. As a quantum field theoretical system, QCD has been extensively applied to situations in which its perturbative content provides a dependable computational tool. It is, in fact, within the framework of this perturbative content that QCD has successfully confronted the quantitative description of the multitude of scattering processes, which probe strong interaction dynamics at high energies. Admittedly, the study of the non-perturbative domain of the theory offers intriguing and, most certainly, fundamental challenges. Nevertheless, the immediate need to confront recent measurements coming from the HERA and Tevatron particle accelerators as well as the expected ones, in the near future, from the LHC accelerator continues to put perturbative QCD (pQCD) at the forefront of theoretical activity.

Given the non-abelian structure of QCD, the (by far) most demanding component of the theory, with respect to perturbative calculations, is its gluonic, as opposed to its quark, sector $^{1}$. In particular, perturbative computations involving Feynman diagrams with gluon/ghost loops become, to say the least, quite monstrous. During the last decade or so various methods, aiming to expedite Feynman diagram computations in QCD, have been proposed whose basic feature is that they rely in a first, rather than the usual second, quantization approach to the formulation of the theory. Corresponding attempts have employed either strings [1-3], or world-line paths [4-11] as their underlying basic agents. Within the framework of the latter case, two of the present authors $[10,11]$, have been involved in work which led to the formulation of a set of master expressions, that condense the multitude of all Feynman diagrams entering a given configuration. These expressions are determined by the number of loops and the number of external gluon propagators attached to them. To be more precise, the derived expressions go up to two loop configurations, nevertheless the "logic" of the construction can be extended to loops of higher order. Suffice it to say, at this point, that the analytical confrontation of a two loop situation with four "external" gluon lines constitutes a challenging enough problem [12].

\footnotetext{
${ }^{1}$ For that matter, this is more so the case for the non-perturbative domain of the theory.
} 
The basic feature of the master expressions arrived at in $[10,11]$ is that they are furnished in terms of a set of Grassman and a set of Feynman variables. Once integrations over these two sets of variables are performed one obtains the full result, i.e. the one which, for the given configuration, contains the contribution of all Feynman diagrams at once. It is obvious, even before laying an eye on these master formulas, that in order to put them into practical use, their confrontation calls for the employment of suitable computational methods. It is the aim of this paper to present a program, which will be applied to the one gluon loop case for $M$ external gluonic lines ( $M$ fairly large). The main part of our program deals with the confrontation of multi-Grassmann variable integrals and arrives at expressions which involve the appropriate set of Feynman parameters only. This program could hopefully find applicability to other situations, where multi-Grassmann variable integrals also make their entrance.

Our paper is organized as follows. In the following section we present the battery of formulas, which are associated with the master expression corresponding to one gluon/ghost loop with $M$ external gluon attachments in all possible ways. We shall consider cases up to fairly large values of $M$, which exhibit divergent terms ${ }^{2}$, in addition to finite ones. In section 3 we present the ideas on which this program is based along with the formulas which count the terms appearing in our final result. In section 4 we describe the structure of the program, while section 5 presents our results, accompanied by tables and figures. Finally, our concluding remarks are made in section 6 .

\section{The one loop master formula}

Consider a configuration consisting of one gluon/ghost loop onto which $M$ external gluon lines, with corresponding momenta $p_{1} \cdots p_{M}$ are attached (see Figure 1). According to [10], the master expression, which summarizes the total contribution from all Feynman diagrams pertaining to this configuration is given by

$\Gamma_{1}^{(M)}\left(p_{1}, \ldots, p_{M}\right)=-\frac{1}{2} g^{M}(2 \pi)^{4} \delta^{(4)}\left(\sum_{n=1}^{M} p_{n}\right) \operatorname{Tr}_{C}\left(t_{G}^{\alpha_{M}} \cdots t_{G}^{\alpha_{1}}\right) \frac{1}{(4 \pi)^{2}} \int_{0}^{\infty} d T T^{M-3} \times$

\footnotetext{
${ }^{2}$ As expected, the aforementioned master expressions implicate the absence of divergent terms for $M>4$, cf. [10].
} 


$$
\begin{aligned}
& \times\left[\prod_{n=M}^{1} \int_{0}^{1} d u_{n}\right] \theta\left(u_{M}, \ldots, u_{1}\right) F^{(M)}\left(u_{1}, \ldots, u_{m} ; T\right) \exp \left[T \sum_{n<m} p_{n} \cdot p_{m} G\left(u_{n}, u_{m}\right)\right] \\
& \quad+\text { permutations }
\end{aligned}
$$

where $g$ is the coupling constant of the theory, the $t_{G}^{\alpha_{i}}, i=1, \ldots, M$ are the $S U(3)_{\text {color }}$ group generators (in the adjoint representation) with $\operatorname{Tr}_{C}$ the trace over the color group, the $u_{i}$ are Feynman parameters, the function $\theta$ is specified by

$$
\theta\left(u_{M}, \ldots, u_{1}\right)=\theta\left(u_{M}-u_{M-1}\right) \ldots \theta\left(u_{2}-u_{1}\right)
$$

and

$$
\begin{aligned}
& F^{(M)}\left(u_{1}, \ldots, u_{M} ; T\right)=\left[\prod_{n=M}^{1} \int d \xi_{n} d \bar{\xi}_{n}\right]\left(\operatorname{Tr}_{L} \Phi^{[1]}-2\right) \times \\
\times & \exp \left[\sum_{n} \sum_{m \neq n} \bar{\xi}_{n} \xi_{n} \varepsilon^{n} \cdot p_{m} \partial_{n} G\left(u_{n}, u_{m}\right)+\frac{1}{2 T} \sum_{n} \sum_{m \neq n} \bar{\xi}_{n} \xi_{n} \bar{\xi}_{m} \xi_{m} \varepsilon^{n} \cdot \varepsilon^{m} \partial_{n} \partial_{m} G\left(u_{n}, u_{m}\right)\right] .
\end{aligned}
$$

In the above relation the $\xi$ 's are Grassmann variables, the $\varepsilon^{i}$ are polarization vectors for the external gluons, $\Phi^{[1]}$ is the so-called spin factor entering the world-line description of QCD (see below), with $\operatorname{Tr}_{L}$ denoting trace with respect to Lorenz generator representation indices and the $G\left(u_{n}, u_{m}\right)$ are free propagators for the particle modes entering the worldline path integral description of QCD, in the context of its first quantized version (see [10]). They obey the equation(s)

$$
-\partial_{n} \partial_{m} G\left(u_{n}, u_{m}\right)=\partial_{n}^{2} \dot{G}\left(u_{n}, u_{m}\right) \equiv \ddot{G}\left(u_{n}, u_{m}\right)=2\left[\delta\left(u_{n}, u_{m}\right)-1\right]
$$

with boundary condition

$$
\partial_{n} G\left(u_{n}, u_{m}\right) \equiv \dot{G}\left(u_{n}, u_{m}\right)=\operatorname{sign}\left(u_{n}-u_{m}\right)-2\left(u_{n}-u_{m}\right)=-\dot{G}\left(u_{m}, u_{n}\right) .
$$

The explicit expression for the spin factor in terms of the set of parameters entering our expressions is (the $J_{\mu \nu}$ are the Lorentz generators, in the vector representation)

$$
\begin{aligned}
& \Phi_{\mu \nu}^{[1]}=P \exp \left[\frac{i}{2} \sum_{n=1}^{M} J \cdot \phi(n)\right]_{\mu \nu}= \\
& =\delta_{\mu \nu}+\frac{i}{2}\left(J_{\rho \sigma}\right)_{\mu \omega} \sum_{n=1}^{M} \phi_{\rho \sigma}(n)+\left(\frac{i}{2}\right)^{2}\left(J_{\rho_{2} \sigma_{2}}\right)_{\mu \lambda}\left(J_{\rho_{1} \sigma_{1}}\right)_{\lambda \nu} \sum_{n_{2}=1}^{M} \sum_{n_{1}=1}^{n_{2}-1} \phi_{\rho_{2} \sigma_{2}}\left(n_{2}\right) \phi_{\rho_{1} \sigma_{1}}\left(n_{1}\right)+\ldots
\end{aligned}
$$


where

$$
\phi_{\mu \nu}(n)=2 \bar{\xi}_{n} \xi_{n}\left(\varepsilon_{\mu}^{n} p_{n, \nu}-\varepsilon_{\nu}^{n} p_{n, \mu}\right)+\frac{4}{T} \bar{\xi}_{n+1} \xi_{n+1} \bar{\xi}_{n} \xi_{n}\left(\varepsilon_{\mu}^{n+1} \varepsilon_{\nu}^{n}-\varepsilon_{\nu}^{n+1} \varepsilon_{\mu}^{n}\right) \delta\left(u_{n+1}-u_{n}\right)
$$

A point of note is the following: In the above expressions a specific time ordering has been chosen according to which index $n+1$ comes immediately after index $n$, with $\xi_{M+1}=$ $\bar{\xi}_{M+1}=0$.

The saturation of indices $\rho, \sigma$ in $J_{\rho \sigma}$ is performed instantly, since

$$
\begin{gathered}
(i / 2)\left(J_{\rho \sigma}\right)_{\mu \nu} 2\left(\varepsilon_{\rho}^{n} p_{n, \sigma}-\varepsilon_{\sigma}^{n} p_{n, \rho}\right)=-2\left(\varepsilon_{\mu}^{n} p_{n, \nu}-\varepsilon_{\nu}^{n} p_{n, \mu}\right), \\
(i / 2)\left(J_{\rho \sigma}\right)_{\mu \nu}(4 / T)\left(\varepsilon_{\rho}^{n+1} \varepsilon_{\sigma}^{n}-\varepsilon_{\sigma}^{n+1} \varepsilon_{\rho}^{n}\right)=-(4 / T)\left(\varepsilon_{\mu}^{n+1} \varepsilon_{\nu}^{n}-\varepsilon_{\nu}^{n+1} \varepsilon_{\mu}^{n}\right)
\end{gathered}
$$

Thus, eq. (5) may be rewritten as

$$
\Phi_{\mu \nu}^{[1]}=\delta_{\mu \nu}+(-1) \sum_{n=1}^{M} \phi_{\mu \nu}(n)+(-1)^{2} \sum_{n_{2}=1}^{M} \sum_{n_{1}=1}^{n_{2}-1} \phi_{\mu \lambda}\left(n_{2}\right) \phi_{\lambda \nu}\left(n_{1}\right)+\ldots .
$$

\section{Theoretical considerations surrounding the computation}

The object of computation is the quantity $F^{(M)}$, as given by eq. (2). The main effort amounts to carrying out the Grassmann integrations entering this expression. In between there intervenes the task of performing trace operations over Lorentz indices associated with the $\operatorname{loop}(\mathrm{s})$. The tracing involves strings of the $\phi_{\mu \nu}(n)$, cf. eq. (6). The Lorentz trace can be postponed until after the Grassmann integration. This trace accounts for setting the first and the last Lorentz index in a series of products of the objects $\phi_{\mu \nu}(n)$ equal. Since the second index of a $\phi_{\mu \nu}(n)$ factor must be saturated with first index of the factor that follows, the Grassmann integrations will be carried out first if the specific order by which the sequence of the $\phi_{\mu \nu}(n)$ 's are placed in each product is kept undisturbed. Accordingly, the $\phi_{\mu \nu}(n)$ are considered non-commutative objects during the Grassmann integration. By activating this rule the Lorentz indices can be dropped until after the Grassmann integration has been completed.

To get a concrete handle on the situation, we introduce the quantities

$$
A_{n}=-2\left(\varepsilon_{\mu}^{n} p_{n, \nu}-\varepsilon_{\nu}^{n} p_{n, \mu}\right)
$$




$$
\begin{gathered}
B_{n}=-\frac{4}{T}\left(\varepsilon_{\mu}^{n+1} \varepsilon_{\nu}^{n}-\varepsilon_{\nu}^{n+1} \varepsilon_{\mu}^{n}\right) \delta\left(u_{n+1}-u_{n}\right), \\
C_{n}=\sum_{m \neq n} \varepsilon^{n} \cdot p_{m} \partial_{n} G\left(u_{n}, u_{m}\right)
\end{gathered}
$$

and

$$
D_{n m}=\frac{1}{2 T} \varepsilon^{n} \cdot \varepsilon^{m} \partial_{n} \partial_{m} G\left(u_{n}, u_{m}\right) .
$$

Using the fact that for commutative objects $\exp (C+D)=\exp (C) \exp (D)$, the Grassmann calculation assumes the form

$$
\begin{gathered}
F^{(M)}=\left[\prod_{n=M}^{1} \int d \xi_{n} d \bar{\xi}_{n}\right]\left\{P \exp \left[\sum_{n}\left(\bar{\xi}_{n} \xi_{n} A_{n}+\bar{\xi}_{n+1} \xi_{n+1} \bar{\xi}_{n} \xi_{n} B_{n}\right)\right]-2\right\} \\
\exp \left[\sum_{n} \bar{\xi}_{n} \xi_{n} C_{n}\right] \exp \left[\sum_{n} \sum_{m \neq n} \bar{\xi}_{n} \xi_{n} \bar{\xi}_{m} \xi_{m} D_{n m}\right] .
\end{gathered}
$$

A direct way to proceed with this calculation is to suitably code the functions and the Grassmann variables and then separately calculate the objects $\exp \left[\sum_{n} \bar{\xi}_{n} \xi_{n} C_{n}\right]$ $\exp \left[\sum_{n} \sum_{m \neq n} \bar{\xi}_{n} \xi_{n} \bar{\xi}_{m} \xi_{m} D_{n m}\right]$ and $\left\{P \exp \left[\sum_{n}\left(\bar{\xi}_{n} \xi_{n} A_{n}+\bar{\xi}_{n+1} \xi_{n+1} \bar{\xi}_{n} \xi_{n} B_{n}\right)\right]-2\right\}$. Each of these objects may contain sums of products with at most $M$ Grassmann variables $\xi_{n}$. Then the multiplication between them can be carried out. Since the following Grassmann properties are valid

$$
\int d \xi_{n} \xi_{n}=1, \int d \bar{\xi}_{n} \bar{\xi}_{n}=1, \int d \xi_{n}=\int d \bar{\xi}_{n}=0, \xi_{n} \xi_{m}=-\xi_{m} \xi_{n}, \bar{\xi}_{n} \bar{\xi}_{m}=-\bar{\xi}_{m} \bar{\xi}_{n}
$$

the output of the integration becomes obvious. Only those terms survive which contain exactly $M$ products of $\xi_{n} \bar{\xi}_{n}$ with all the indices different from each other. This calculation is straightforward and we have written out the corresponding FORTRAN code for carrying it out [13]. The routine is effective (we present in Fig. 3 results up to $M=10$ ), but the time consumed to reach the result rises exponentially with larger values of $M$. The basic obstacle to the effectiveness of the routine is that the plethora of terms involved in the intermediate computations does not survive in the final result. A second source of trouble is the consumed amount of time for rearranging terms at each stage of the computation before identical ones are gathered and summed.

In this paper the aforementioned issues are confronted by following a different computational procedure, which is based on the following objectives: (a) at each stage of the 
calculation only the surviving terms are computed and (b) similar terms are grouped together in the final result. The above goals become more concrete by making the following observations concerning the form of the terms entering our final expressions. These terms will, in general, be products of the quantities $A_{n}, B_{n}, C_{n}$ and $D_{n m}$ accompanied by Grassmann variables the origin of each one of which becomes evident from eq. (14). It is also apparent that each of $A_{n}$ or $C_{n}$ is accompanied by one pair of Grassmann variables, whereas two such pairs accompany $B_{n}$ and $D_{n m}$. Let $N_{1}, N_{2}, N_{3}$ and $N_{4}$ be the number of the functions $A_{n}, B_{n}, C_{n}$ and $D_{n m}$, respectively appearing in a given term of the final result. According to the Grassmann properties, the terms entering this result must satisfy

$$
N_{1}+2 N_{2}+N_{3}+2 N_{4}=M, 0 \leq N_{i} \leq M, N_{3}+N_{4} \neq 1, N_{i} \in \mathbf{N}, i=1,2,3,4,
$$

with the condition $N_{3}+N_{4} \neq 1$ coming from the fact that the Lorentz trace of a single function $A_{n}$ or $B_{n}$ is zero.

The above equation is very important because it helps one to determine all the possible forms of the final terms before actually carrying out the calculation. Every group of the integers $N_{i}$ represents a particular form of the final terms, each of which will be referred to as "structure". Finding all the possible groups of $N_{i}$ suffices to determine all the possible structures. In this way the calculation is broken into a number of subcalculations in each of which one collects all the terms that correspond to a specific structure. A term belonging to a particular structure cannot be similar to a term belonging to another structure, since such terms will contain different numbers of functions $A_{n}, B_{n}, C_{n}$ and $D_{n m}$, so goal (b) is satisfied.

Let us turn now to goal (a). Since in the final result there must exist exactly $M$ pairs of Grassmann variables, all different from each other, it is evident that every part of one exponential entering eq. (14) has to be multiplied by those components of the rest of the exponentials which satisfy eq. (16). We shall try now to trace how many terms survive in a specific structure characterised by the integers $N_{i}$.

Firstly, as far as the $P \exp$ function entering (14) is concerned, one may ask how can one obtain exactly $N_{1}$ functions $A_{n}$ and $N_{2}$ functions $B_{n}$ placed in an ordered product according to a specific time order. It is better to count the terms of the substructure 
$N_{1}, N_{2}$ first because the fact that the associated product is ordered imposes more restrictive conditions. For every group of Grassmann pairs taken from the total $M$ pairs which enter the substructure $N_{1}, N_{2}$, the remaining Grassmann pairs will always form a substructure $N_{3}, N_{4}$ (according to (16)). The converse, however, is not true: If a substructure $N_{3}, N_{4}$ is formed first, then the remaining Grassmann pairs cannot always form a substructure with given $N_{2}$. The reason is that the quantities $B_{n}$ are associated with the Grassmann factor $\bar{\xi}_{n+1} \xi_{n+1} \bar{\xi}_{n} \xi_{n}$, connecting adjacent Grassmann pairs. Consider, for example, the case where $N_{2}=1$ and the time order is of the form $1,2,3, \cdots$. If, now, we are left with the Grassmann indices 1,3 , after considering the previous terms, then it is obvious that such a case will not lead to surviving terms.

Generally speaking there are $\left(\begin{array}{c}N_{1}+N_{2} \\ N_{1}\end{array}\right)$ ways one can have products of $N_{1} A_{n}$ functions and $N_{2} B_{n}$ functions, if one is not concerned with the index $n$ they carry. This result counts the ways the $N_{1} A_{n}$ functions (considered indistinguishable) can occupy $N_{1}+N_{2}$ total vacant places. The rest are then filled with the functions $B_{n}$. It can be checked that for everyone of these products of functions the ways the indices $n$ can be distributed among the functions of the same kind is the same. So let us consider a product where the first $N_{2}$ functions are of the kind $B_{n}$ (contributing $2 N_{2}$ Grassmann pairs) and the last $N_{1}$ functions are of the kind $A_{n}$ (contributing $N_{1}$ Grassmann pairs). If the first $2 N_{2}$ Grassmann pairs (according to the given time order) are attributed to the $N_{2}$ functions $B_{n}$, then there are $M-2 N_{2}$ Grassmann pairs left for the $N_{1}$ functions $A_{n}$ and there are $\left(\begin{array}{c}M-2 N_{2} \\ N_{1}\end{array}\right)$ ways this can be achieved. Let us now suppose that the first $2 N_{2}+1$ Grassmann pairs have been used up through $N_{2}$ functions $B_{n}$. This means that a single Grassmann pair is missing, hence one of the $B_{n}$ should begin by skipping the Grassmann pair that precedes it. There are $N_{2}$ ways this can happen and the result is that there are now $M-2 N_{2}-1$ Grassmann pairs to be distributed among $N_{1}$ places. The whole scheme goes on this way. Each time one uses $2 N_{2}$ from the first $2 N_{2}+i$ Grassmann pairs in the $N_{2} B_{n}$ functions. The ways this can be done are equal to the ways one can distribute $i$ indistinguishable objects at $N_{2}$ distinguishable places. The result is $\left(\begin{array}{c}i+N_{2}-1 \\ N_{2}-1\end{array}\right)$ and has to be multiplied by $\left(\begin{array}{c}M-2 N_{2}-i \\ N_{1}\end{array}\right)$, which 
are the ways the remaining Grassmann pairs can be distributed in the $N_{1}$ places. Summing for all $i$ we have the result

$$
\sum_{i=0}^{M-N_{1}-2 N_{2}}\left(\begin{array}{c}
i+N_{2}-1 \\
N_{2}-1
\end{array}\right)\left(\begin{array}{c}
M-2 N_{2}-i \\
N_{1}
\end{array}\right) .
$$

Eq. (17) is true when $N_{2} \neq 0$. When $N_{2}=0$ the corresponding result is simply the ways we can distribute the $M$ Grassmann pairs in $N_{1}$ places, that is

$$
\left(\begin{array}{c}
M \\
N_{1}
\end{array}\right)
$$

Since the products coming from the $P$ exp function are ordered they are all unique, which means that each one of them registers directly, i.e. without any weight factor.

Our next step is to consider how do products of exactly $N_{3}$ Grassmann pairs $\bar{\xi}_{n} \xi_{n}$ attributed to $\exp \left[\sum_{n} \bar{\xi}_{n} \xi_{n} C_{n}\right]$ arise, if the unused Grassmann pairs from the substructure $N_{1}, N_{2}$ are $\nu_{3}=M-N_{1}-2 N_{2}$. Obviously, the latter products originate from the component of the exponential term

$$
\frac{1}{N_{3} !} \prod_{j=1}^{N_{3}}\left(\sum_{n_{j}=1}^{\nu_{3}} \bar{\xi}_{n_{j}} \xi_{n_{j}} A_{n_{j}}\right)
$$

The sum in the above expression is extended over all the Grassmann pairs that have not been used in the previous substructure, since the entrance of anyone of the rest $M-\nu_{3}$ pairs in this sum will have null contribution to the final result. In short, given that the double appearance of a Grassmann variable in any product causes it to vanish, we need to find the number of terms that survive. This number is equal to the number of the ways the $\nu_{3}$ Grassmann pairs can be distributed in $N_{3}$ places. Each place, here, corresponds to the position the particular Grassmann pair will enter the product. Generally speaking $\nu_{3}$ Grassmann pairs are allowed to occupy the first position. For every Grassmann in the 1st position, $\nu_{3}-1$ Grassmann pairs are allowed to occupy the 2 nd, etc. All the allowed products are therefore the orders of $\nu_{3}$ per $N_{3}$, that is $\left(\nu_{3}\right)_{N_{3}}=\nu_{3}\left(\nu_{3}-1\right) \cdots\left(\nu_{3}-N_{3}+1\right)$. But these terms are not all different. Two Grassmann pairs, given that they contain two Grassmann variables, may change their position without having any effect on their relative sign. So the products of $N_{3}$ pairs are identical if they contain the same Grassmann variables. We may then collect these identical products. Their number coincides with the number of the permutations of $N_{3}$ objects to $N_{3}$ 
places, that is $N_{3}$ !. The number of different terms from the part of the exponential given by (18) diminishes then to combinations of $\nu_{3}$ per $N_{3}$

$$
\begin{gathered}
\frac{\left(\nu_{3}\right)_{N_{3}}}{N_{3} !}=\frac{\nu_{3}\left(\nu_{3}-1\right) \cdots\left(\nu_{3}-N_{3}+1\right)}{N_{3} !}=\frac{\nu_{3}\left(\nu_{3}-1\right) \cdots\left(\nu_{3}-N_{3}+1\right)\left(\nu_{3}-N_{3}\right) !}{N_{3} !\left(\nu_{3}-N_{3}\right) !}= \\
=\left(\begin{array}{c}
\nu_{3} \\
N_{3}
\end{array}\right) .
\end{gathered}
$$

The factor accompanying each of these terms will be then $N_{3}$ !, divided by the same factor entering (19) in the denominator. The result will be an overall unit factor.

Next we ask how do products of exactly $2 N_{4}$ Grassmann pairs $\bar{\xi}_{n} \xi_{n}$ enter through $\exp \left[\sum_{n \neq m} \bar{\xi}_{n} \xi_{n} \bar{\xi}_{m} \xi_{m} D_{n m}\right]$, if the unused Grassmann pairs from the previous substructures are $\nu_{4}=M-N_{1}-2 N_{2}-N_{3}$. These products originate from the part of the exponential

$$
\frac{1}{N_{4} !} \prod_{j=1}^{N_{4}}\left(\sum_{n_{j}=1}^{\nu_{4}} \sum_{m_{j} \neq n_{j}} \bar{\xi}_{n_{j}} \xi_{n_{j}} \bar{\xi}_{m_{j}} \xi_{m_{j}} D_{n_{j} m_{j}}\right) \text {. }
$$

The number of terms that survive is the number of the ways we can distribute the $\nu_{4}$ Grassmann pairs to $2 N_{4}$ positions, since every function $D_{n m}$ provides two positions of Grassmann pairs. The number of allowed products are the orders of $\nu_{4}$ per $2 N_{4}$, that is $\left(\nu_{4}\right)_{2 N_{4}}$. In order to isolate different terms we must divide by the ways the $N_{4}$ functions $D_{n m}$ could appear in the product. There are $N_{4}$ ! such ways. But since every $D_{n m}$ function is equal to a $D_{m n}$ function, we have to divide by a factor of 2 for every such function. We have $N_{4}$ such functions, so the total factor by which we divide is $2^{N_{4}}$. Summarising, the number of surviving, different terms is

$$
\frac{1}{2^{N_{4}} N_{4} !}\left(\nu_{4}\right)_{\left(2 N_{4}\right)}=\frac{\left(2 N_{4}\right) !}{2^{N_{4}} N_{4} !}\left(\begin{array}{c}
\nu_{4} \\
2 N_{4}
\end{array}\right) .
$$

The factor accompanying each of these terms will then be $2^{N_{4}} N_{4}$ !, divided by the $N_{4}$ ! which appears in (21), the net result being an overall factor of $2^{N_{4}}$.

Collecting the above findings we arrive at the conclusion that the total number of terms in a structure $N_{1}, N_{2}, N_{3}, N_{4}$ that survive, while being different, is

$$
R\left(M, N_{1}, N_{2}\right)\left(\begin{array}{c}
N_{1}+N_{2} \\
N_{1}
\end{array}\right)\left(\begin{array}{c}
M-N_{1}-2 N_{2} \\
N_{3}
\end{array}\right) \frac{\left(2 N_{4}\right) !}{2^{N_{4}} N_{4} !}\left(\begin{array}{c}
M-N_{1}-2 N_{2}-N_{3} \\
2 N_{4}
\end{array}\right)
$$


where

$$
R\left(M, N_{1}, N_{2}\right)=\left\{\begin{array}{l}
\sum_{i=0}^{M-N_{1}-2 N_{2}}\left(\begin{array}{c}
i+N_{2}-1 \\
N_{2}-1
\end{array}\right)\left(\begin{array}{c}
M-2 N_{2}-i \\
N_{1}
\end{array}\right), \text { if } N_{2} \neq 0 \\
\left(\begin{array}{l}
M \\
N_{1}
\end{array}\right), \text { if } N_{2}=0
\end{array} .\right.
$$

The accompanying factor for every term is $2^{N_{4}}$.

The above considerations are important in the building up of the program. First the knowledge of the factor accompanying each term is necessary, since in this way one avoids producing identical terms and subsequently summing them up. Also the number of different terms existing in each substructure is needed to parameterise the dimensions of the arrays that are used in the communication between the subroutines of the program, as it will become apparent in the next section. Moreover, the theoretical prediction of the number of terms expected in the final result is used to verify the output of the program. This is most important especially for large $M$, where there are numerous surviving terms.

\section{Presenting the structure of the program}

The program has been written in FORTRAN 90. The reason for this choice is the use of allocatable arrays in all the subroutines. This enables us to parameterise the dimensions of these arrays for different values of $M$, since the size of some of them grows drastically with $M$. We are also able to manage the available memory effectively.

According to our previous analysis we shall first produce in the program all the groups of integers $N_{1}, N_{2}, N_{3}, N_{4}$ that define via eq. (16) the compatible structures for given $M$. This is accomplished in the subroutine STRUCTUREMATRIX, which performs four loops at which the integers $N_{1}$ and $N_{3}$ acquire all values from 0 to $M$ and the integers $N_{2}$ and $N_{4}$ all values from 0 to $M / 2$. Once a successful combination for the values of these integers is found, according to (16), it is copied to a temporary matrix, for which its column dimension is known a priori. This dimension is the number of all the different structures that exist for a particular $M$, a number that grows relatively slowly with $M .{ }^{3}$ This number can be easily evaluated for all $M$ and has been inserted to the data of the program for all $M \leq 20$. For

\footnotetext{
${ }^{3}$ For example it equals 486 for $M=20$.
} 
$M>20$ the program calls, where it is needed, subroutine DIMOFSTRUCTUREMATRIX, which produces the number of different structures for the given $M$. Given that we are interested in grouping the resulting terms according to their power of $T$, we group the structures according to this power. It is easily checked from eqs. (1), (11) and (13) that this power is $M-3-N_{2}-N_{4}$. The final result is inserted into a matrix which is the output of STRUCTUREMATRIX.

The surviving terms in the final result are produced in subroutine MULTIPLYALL. The latter first calls subroutine STRUCTUREMATRIX and then for every particular structure it calculates first the compatible substructure $N_{1}, N_{2}$, followed by the compatible substructure $N_{3}$ and finally the compatible substructure $N_{4}$.

Three complementary routines to MULTIPLYALL have been constructed. The first is subroutine COMBINATIONASCEND, which produces all the combinations of the $L$ natural numbers from 1 to $L$ in groups of $K$ and places them in ascending order in the lines of a matrix which constitutes the output of the subroutine. An array $A$ with $K$ elements is used for temporary inputs. The numbers from 1 to $K$ are placed in the first line of the output matrix, with the last $K$-th column being marked as the change column. This means that the lines which follow in the output matrix are written with the first $K-1$ elements the same while the $K$-th element acquires all the values from $K+1$ to $L$. The routine is repeated with the change column lowered by one. If the change column is the $J$-th, it is checked whether

$$
A_{J} \leq M-N+J .
$$

If (25) holds, the elements with index greater than $J$ acquire the values

$$
A_{J+I}=A_{J}+I,(I=J+1, K) .
$$

Then the array $A$ is placed in the output and $K$-th column is marked again as the change column, repeating the procedure. In the opposite case, where (25) is false, the change column is lowered by one, until condition (25) is fulfilled. An example of the output of this subroutine is given in Table 1.

The second subroutine is ORDERS, which finds the orders of $L$ natural numbers from 1 to $L$ in groups of $K$ and places them in the lines of the output matrix $C$. The matrix 
$C$ is filled column by column. In every column two integers determine the filling course: The first is the number of identical elements of the previous column. This integer is called STEPGREAT and for the $i$-th column it equals $\frac{(L-i+1) !}{(L-K) !}$. This expression gives the number of the orders of the $L-i+1$ numbers which are left after the first $i-1$ have correspondingly occupied the first $i-1$ columns. The second integer, called STEPSMALL, is the number of repetitions of the same element in the $i$-th column and equals STEPGREAT divided by $L-i+1$. An example of the gradual filling of matrix $C$ is shown in Table 2 .

The third subroutine is COMBINATIONPERTWO which finds the combinations of the $L^{\prime}$ natural numbers from 1 to $L^{\prime}$ in groups of $K^{\prime}$ after they have been grouped in couples. Each group is placed in ascending order in the output matrix $C$. Each couple always enters as $n m$ with $n<m$. Matrix $C$ occupies $2 K^{\prime}$ columns so that each couple is located at adjacent columns. To achieve this COMBINATIONPERTWO calls first COMBINATIONASCEND (with $L=L^{\prime}$ and $K=K^{\prime}$ ) and stores the result in matrix $C A$. Then it calls ORDERS (with $L=L^{\prime}-K^{\prime}$ and $K=K^{\prime}$ ) and stores the result in matrix $C B$. Matrix $C A$ produces the odd columns of matrix $C$, that is the first elements of the couples. For every line of $C A$ the remaining $L^{\prime}-K^{\prime}$ elements are found and placed in a temporary array $B$. The second element of every couple is found from matrix $C B$ after its elements are read as indices of the array $B$. Because of this last action subroutine ORDERS is only called once in COMBINATIONPERTWO. In the production of the group of couples it is checked whether the second element is greater than the first. If this is not so the subroutine proceeds to the next combination. An example of the output of the subroutine is given in Table 3.

With the three complementary subroutines that have been described above the calculation of the substructures can be carried out. First the calculation of the substructure $N_{1}, N_{2}$ is produced. By calling COMBINATIONASCEND from MULTIPLYALL the combinations of the positions of the $N_{2} B_{n}$ functions from a total of $N_{1}+N_{2}$ vacancies are found and placed in the matrix CPL. For every such combination, which is represented as a line of $\mathrm{CPL}$, one has to produce all the compatible products of functions $A_{n}$ and $B_{n}$. First we shall assume that the integers $n$, which characterise the functions $A_{n}$ and $B_{n}$, run from 1 to $M$ and that they must appear in an ascending order, from small to large numbers. We then allocate two matrices CTEMP1 and CTEMP2 with equal dimensions. The number 
of columns equals $N_{1}+N_{2}$ and the number of lines is given by eq. (17) or (18). The last value is returned by function COUNTTERMS2. For every element in the positions from 1 to $N_{1}+N_{2}$ the maximum and minimum allowed values are found and are placed in the arrays IMAX and IMIN respectively. One has to bear in mind that every function $B_{n}$ consumes two consequent integers, so when one such function, characterised by integer $n$, appears in the $i$-th place, then the integer in the $(i+1)$-th place cannot be smaller than $n+2$. The calculation begins by inserting in the first column of matrix CTEMP1 and in the different lines all the allowed values for the first integer, as they are dictated by the arrays IMAX and IMIN. In the second run all the allowed combinations of the first two elements will be copied in matrix CTEMP2. To this end, for each line of CTEMP1 the allowed values of the next element are determined. These allowed values can range from the maximum to the minimum value of the arrays IMAX and IMIN but they must also fulfil additional constraints which depend on the preceding integer. If the preceding integer is $n$, then the next integer will be $n+1$ when the corresponding function is of the type $A$ and $n+2$ if it is of type $B$. So each line of CTEMP1 does not necessarily correspond to the same number of lines of CTEMP2. The calculation proceeds with the combinations of the three first elements copied back to CTEMP1 with the data taken from CTEMP2 and so forth. Once all the elements are exhausted, their allowed combinations will have been accommodated in one of the matrices CTEMP1 or CTEMP2 and are then copied in matrix C12. In the following example we suppose that $M=5, N_{1}=2, N_{2}=1$ and that the lone function $B$ occupies the middle place among the three available for the functions $A$ and $B$. The array IMIN has, subsequently, acquired the values $\left(\begin{array}{lll}1 & 2 & 4\end{array}\right)$ and the array IMAX the values $\left(\begin{array}{lll}2 & 3 & 5\end{array}\right)$. The calculation of the allowed combinations proceeds as follows

$$
\left(\begin{array}{c}
1 \\
2
\end{array}\right) \rightarrow\left(\begin{array}{ll}
1 & 2 \\
1 & 3 \\
2 & 3
\end{array}\right) \rightarrow\left(\begin{array}{lll}
1 & 2 & 4 \\
1 & 2 & 5 \\
1 & 3 & 5 \\
2 & 3 & 5
\end{array}\right)
$$

The compatible substructure $N_{3}$ for every line in the substructure $N_{1}, N_{2}$ is produced with the call of the subroutine COMBINATIONASCEND with $L=M-N_{1}-2 N_{2}$ and 
$K=N_{1}$ and the output is stored in matrix $C 1$. Also, for every line of CPL the remaining elements which have not been used are determined and placed in the array LEFT34. Matrix $C 1$ contains the indices of the array LEFT34, i.e. the presence of 2 in a line of $C 1$ has the meaning of the 2nd element of array LEFT34. With this representation the subroutine COMBINATIONASCEND is called only once from MULTIPLYALL to produce the substructure $N_{3}$.

The compatible substructure $N_{4}$ for every line in the substructures $N_{1}, N_{2}$ and $N_{3}$ is produced with the call of the subroutine COMBINATIONPERTWO with $L^{\prime}=M-N_{1}-$ $2 N_{2}-N_{3}$ and $K^{\prime}=N_{4}$ and the output is stored in matrix $C 2$. Also for every line of CPL and every line of $\mathrm{C} 1$ the remaining elements which have not been used are found and placed in the array LEFT4. Matrix $C 2$ contains the indices of the array LEFT4, i.e. the presence of 2 in a line of $C 2$ has the meaning of the 2nd element of array LEFT4. With this representation the subroutine COMBINATIONPERTWO is called only once from MULTIPLYALL to produce the substructure $N_{4}$.

Until now we have described the production of the sequence of functions $A, B, C$ and $D$ in the terms that survive in the final result. The indices $n$ which characterise the functions in this description are considered as natural numbers from 1 to $M$ and their order is considered to be an ascending one. Clearly, the order of these natural numbers is the time order given a priori by the user. This time order is stored in the array TOR. So in order to obtain the final result we have to read the elements in the structure which has been described as indices of the array TOR. The output of MULTIPLYALL is, for every structure, a series of lines which represent the products of the functions (10)-(13). Their representation, which is given by integers, is listed in Table 4. It should be noted that even though the functions $A_{n}, B_{n}$ and $D_{n m}$ may happen to be represented by the same integer, they cannot be confused, because they have a specific order of appearance. Specifically the non-commutative objects of the substructure $N_{1}, N_{2}$ appear first, followed by the commutative objects of the structure $N_{3}$ and finally by those of the structure $N_{4}$.

The output of MULTIPLYALL is written in a series of files with the first one designated as F001. When a file exceeds 18,000,000 terms then the next structure will be written in the next file. Each line in a structure is accompanied by a factor which is just $2^{N_{4}}$, according to 
the discussion of the previous section. In the cases for which the substructure $N_{1}=N_{2}=0$ enters, this factor is multiplied by 2 . This comes from $\operatorname{Tr}_{L}\left(\delta_{\mu \nu}\right)-2=4-2=2$. The output of MULTIPLYALL is written in the form of integers and so it can be used for further calculations by the user.

The program gives in the output of MULTIPLYALL the products of surviving functions. However the Lorentz trace has yet to be performed. This trace involves all the functions in the $N_{1}, N_{2}$ substructure and this is the reason these objects have been considered as being non commutative. The Lorentz indices $\mu, \nu$ in the functions (10), (11) have been considered indefinite, that is they have not been defined at this point by the number which represents the function. So when groups of these types of functions are found together it is assumed that the Lorentz trace has to be performed, according to the exact order by which the corresponding functions appear. The following example illustrates the "translation" of a typical product of three such functions

$$
202301204 \rightarrow(202)_{\mu \rho}(301)_{\rho \sigma}(204)_{\sigma \mu} \rightarrow\left(A_{2}\right)_{\mu \rho}\left(B_{1}\right)_{\rho \sigma}\left(A_{4}\right)_{\sigma \mu}
$$

In order to perform the Lorentz trace and arrive at the final output we have constructed the subroutine TRACE. For the computation of the trace only the part $\left(\varepsilon_{\mu}^{n} p_{n, \nu}-\varepsilon_{\nu}^{n} p_{n, \mu}\right)$ from functions $A_{n}$ and the part $\left(\varepsilon_{\mu}^{n+1} \varepsilon_{\nu}^{n}-\varepsilon_{\nu}^{n+1} \varepsilon_{\mu}^{n}\right)$ from functions $B_{n}$ need to be considered. The following representation is used

$$
\varepsilon_{\nu}^{n} \rightarrow 300+n, \quad p_{n, \nu} \rightarrow 200+n
$$

where $n$ is a two digit integer. Then the relevant part of the functions $A_{n}$ and $B_{n}$ for the trace is represented by two matrices

$$
\begin{gathered}
\varepsilon_{\mu}^{n} p_{n, \nu}-\varepsilon_{\nu}^{n} p_{n, \mu} \rightarrow\left(\begin{array}{r}
1 \\
-1
\end{array}\right)\left(\begin{array}{cc}
300+n & 200+n \\
200+n & 300+n
\end{array}\right), \\
\varepsilon_{\mu}^{n+1} \varepsilon_{\nu}^{n}-\varepsilon_{\nu}^{n+1} \varepsilon_{\mu}^{n} \rightarrow\left(\begin{array}{r}
1 \\
-1
\end{array}\right)\left(\begin{array}{cc}
300+n+1 & 300+n \\
300+n & 300+n+1
\end{array}\right) .
\end{gathered}
$$

In a product of functions $A_{n}$ and $B_{n}$, where the Lorenz indices are active, the second index of one function is saturated with the first index of the next. This continues until the last 
function in the product is reached, whereupon its second index is saturated by the first index of the first function. To accomplish this in the representation (29)-(30) it suffices to perform internal products between the elements of the 2 nd column of the $2 \times 2$ matrix that represents one function and the 1st column of the $2 \times 2$ matrix representing the next function. The elements of the 2 nd column of the last matrix are coupled with the elements of the 1st column of the first. The procedure has been appropriately parameterised in the program to exhaust all the combinations.

The rule $\varepsilon^{n} \cdot p_{n}=0$ is also taken into account. On the other hand, we have not applied the replacement associated with momentum conservation, since it spoils the symmetry of the terms belonging to the same structure in addition to increasing the number of terms, especially for large $M$.

The result of the trace, for each term, corresponds to the sums of products of internal products of $\varepsilon$ 's and $p$ 's. These sums are again represented by series of integers. An internal product is represented by a six digit integer, according to the prescription of Table 5 .

The factor of a product is represented by an integer less than or equal to 200000. Such factors also signal both the beginning of the product of internal products, as well as, its ending. This can be summarised in the next example

$$
\begin{aligned}
& \begin{array}{llllllllll}
-1 & 304301 & 1 & 304303 & 302203 & 301202 & 204204 & -3 & 304203 & 303302
\end{array} \rightarrow \\
& -\varepsilon^{4} \cdot \varepsilon^{1}+\varepsilon^{4} \cdot \varepsilon^{3} \quad \varepsilon^{2} \cdot p_{3} \quad \varepsilon^{1} \cdot p_{2} \quad p_{4} \cdot p_{4}-3 \quad \varepsilon^{4} \cdot p_{3} \quad \varepsilon^{3} \cdot \varepsilon^{2} .
\end{aligned}
$$

The powers of 2 and $T$ associated with the functions (10), (11) and (12) are incorporated into the factor of every term. The representation of the remaining functions are given through integers according to Table 6.

The output of TRACE is registered in two series of files. The first series begins with file G001 and it contains the result of surviving terms grouped according to the structure they belong and are represented through integers according to Tables 5 and 6 . The content of these files, as it is in integer form, may be used for further processing by the user. The second series begins with file H001 and it contains the surviving terms in function-like form. The terms entering files G001 and H001 correspond to the terms stored in files F001, and so on. There is, also, the option to produce with TRACE only the first term of each structure. In that 
case TRACE avoids reading through the whole series of terms produced by MULTIPLYALL. Instead it calls MULTIPLYALL again and asks for the production of only the first term of each structure. MULTIPLYALL writes the result in only one file, FF001, which is then read by TRACE. With this procedure the timesaving in TRACE is enormous.

The different structures that exist for the current $M$ are listed in file STRLOG, along with information about the files used for the output and the consumed time for the run of the program.

\section{Results}

At the beginning of its run the program asks the user to insert the number of external gluons $M$. Because the output of subroutine MULTIPLYALL occupies storage space which increases rapidly with $M$, the user is warned about the approximated space that will be required. This space has been measured from the output of the program until some value of $M$. For greater values of $M$ it is extrapolated through a fitting function of the form $y=\exp \left[a(\ln (x))^{2}+b \ln (x)+c\right]$, where $y$ is the storage space, $x$ the total number of surviving terms and $a, b$ and $c$ free parameters which have been determined through a fit on the existing data. The same function has been used for the extrapolation on the storage space required by TRACE. Then the program requests for the specific time order for which the calculation will be carried out and a check is performed on the correct input of this time order.

Because the output of subroutine TRACE occupies large storage space the user has the option to ask for the full result of this subroutine, or for the calculation of only the first term of every structure, or to skip the subroutine entirely. Each choice of output of TRACE is accompanied by a warning about the required storage space.

In Table $7 \mathrm{a}$ we show the result of MULTIPLYALL for $M=2$ and in Table $7 \mathrm{~b}$ the result of TRACE in function-like form for the same value of $M$. The 23 terms for $M=3$ are shown in function-like form in Table 8. The number of surviving terms grows rapidly when $M$ increases (for $M=15$ the surviving terms are 430,576,126).

In Figure 2 we have plotted the number of surviving terms (left axis - curve (a)) against $M$. In the same figure we have plotted the consumed storage space for the output of MULTI- 
PLYALL (right axis - curve (b)), which is more compact than the full final result of TRACE. At $M=15$ this space grows to about 22.2 Gbytes, making difficult for us to have the full result written on our hard disc for greater $M$ 's.

In Fig. 3 we have plotted the number of structures as a function of $M$ (left axis - curve (a)). On the same graph we have depicted the average number of surviving terms per structure as a function of $M$ (right axis - curve (b)). It is seen that the number of different structures does not grow rapidly, in contrast to the surviving terms per structure. So the time needed for the completion of the program is mainly due to the intense growth of number of these terms.

In Fig. 4 we have plotted the consumed time for the completion of the calculation of MULTIPLYALL (full line) against $M$ (curve (a)). The consumed time is the most crucial parameter since it exhibits the capabilities of our program, especially for large $M$. This is only 2.74 seconds for $M=10$ and 1.75 hours for $M=15$ (on INTEL CELERON 1.8GHz). The capability of the current program is more evident if it is compared with the older program we had developed in [13] and where the calculation is carried out in the straightforward way without the use of structures. With respect to that program we have made the substitution of eq. (12) in order to group functions with the same Grassmann part in the same overall function, as the case is for the present program and have recorded the time consumed for the calculation without the subroutine TRACE. This allows a comparison to be carried out on the same footing. The result is the dotted line (curve (b)). The slashed line in Fig. 4 (curve (c)) is the consumed time in our current program for MULTIPLYALL to be completed but with suppressed writing in the output files which occupy enormous storage space. In this way we carry calculations for greater values of $M$. It is also evident, from the comparison of curves (a) and (c) that a large fraction of the consumed time for MULTIPLYALL is due to the output. In curves (a)-(c) we have used only the time consumed in MULTIPLYALL and have not added the time consumed in TRACE because the larger part of the time in the latter is consumed in writing the output in the appropriate files and not in the actual calculation. The consumed time in TRACE as function of $M$ is presented by curve (i) for the full result and by curve (ii) for the result with only one term per structure.

In Fig. 5 we have plotted the consumed time, as in Fig. 4, but against the surviving 
terms. The consumed time (full line) in our new program as a function of the surviving terms can be fitted through a linear function. The consumed time in our old program grows much more rapidly as function of the surviving terms (dotted line). In that case it can be fitted by a second grade polynomial which has been extrapolated to larger values of $M$. This fitting procedure leads to a value of about 1910 years for $M=15$ and can be compared to the 1.75 hours for the same result with our new program. The difference is due to the appearance in the intermediate calculations of our old program of a vast number of terms which cancel in the final result.

In the overall result divergent terms enter only for $M \leq 4$. These terms have been compared with the analytic calculations for the divergent terms for $M=2, M=3$ and $M=4[10]$ and have been found in total agreement. Also the number of surviving terms according to the program have been compared with the theoretical formulas (23)-(24) and have been found equal. The number of surviving terms between the old and the new program have also been found in agreement.

The methods used for the computation of the master formulas do not place an upper bound on the value of $M$ for the calculations. In the specific program we have written the different representations through integers assuming that $M$ is a two digit integer. The limitations are imposed mainly by the storage space and of course, unavoidably, by the necessary time which, even for linear growth, will reach high values for increasing $M$ 's. The consumed time, though, is expected to shrink proportionally to the increase of the speed of the processor to be used. Another limitation arises from the maximum integer that can be encoded in the processor one uses for the running of the program. This sets an upper bound on the number of the elements that can be allocated to a certain array. The size of the matrix that grows more rapidly is the output of COMBINATIONPERTWO which is called from MULTIPLYALL to produce the substructure $N_{4}$. In processors that use 4-byte integers overflow is expected to arise for $M \geq 20$, while in processors that use 8-byte integers the overflow is expected to appear for $M \geq 34$. It is possible to improve these values of $M$, in any case, by defining the respective array in a more complicated way, but we have avoided doing so, since the considered values of $M$ are already quite high. 


\section{Concluding remarks}

In this paper a computational algorithm has been presented aiming towards calculation of one-gluon loop Feynman diagrams, including ghost loop contributions, with $M$ external gluon attachments, on the basis of the master formulas derived in [10]. Compared with our previous attempt [13], the reasoning of the calculation has been completely changed, resulting to a different algorithm. The basic innovations are the grouping of similar terms together and the dramatic reduction of computation time, thus allowing the calculation for quite large values of $M$. All this being said, there remains, of course, the problem of carrying out the integrations over the Feynman parameters, a task which is challenging in itself, both on the analytical and the numerical front. With respect to the latter, it would be of interest to assess the extent to which recent, relevant, considerations [14] can be applied for the succesful completion of the task, at least up to some reasonably high value of $M$, whose Grassmann variable integration part was accomplished in this work.

Finally, one may express the hope that the particular feature of the constructed algorithm, namely the ability to expedite integrations over a multivariable set, a subset of which is Grassmannian, could find wider applications to analogous situations that may arise in other physical problems wherein Grassmann variables make their entrance. Within the context of the present application, it would be of interest to apply the particular algorithm developed in this work to the two-gluon loop $M=4$ case, the corresponding master expressions for which have been derived in [11]. As a first attempt, one could restrict the relevant computation to the divergent term associated with the $M=2$ configuration and verify the consistency with second order corrections to the running coupling constant in pQCD.

\section{Acknowledgement}

A. I. K. and C. N. K. acknowledge the financial support through the program "Pythagoras

I" (grant no. 016) and from the General Secretariat of Research and Technology of the University of Athens.

\section{References}

[1] Z. Bern and D. A. Kosower, Nucl. Phys. B 321 (1989)605; B 379 (1992)451. 
[2] Z. Bern, L. J. Dixon and D. A. Kosower, Phys. Rev. Lett. 70 (1993)2677 arXiv:hep-ph/9302280.

[3] P. Di Vecchia, L. Magnea, A. Lerda, R. Russo and R. Marotta, Nucl. Phys. B 469 (1996)235 arXiv:hep-th/9601143.

[4] M. J. Strassler, Nucl. Phys. B 385 (1992)145 arXiv:hep-ph/9205205.

[5] M. Reuter, M. G. Schmidt and C. Schubert, Annals Phys. 259 (1997)313 arXiv:hep-th/9610191.

[6] C. Schubert, Phys. Rep. 355 (2001)73 arXiv:hep-th/0101036.

[7] H-T. Sato and M. G. Schmidt, Nucl. Phys. B 524 (1998)742; ibid. 560 (1999)551.

[8] H-T. Sato, M. G. Schmidt and Claus Zahlten, Nucl. Phys. B 579 (2000)492 [arXiv: hep-th/0003070.

[9] A. I. Karanikas and C. N. Ktorides, JHEP 9911 (1999)033 arXiv:hep-th/9905027.

[10] S. D. Avramis, A. I. Karanikas and C. N. Ktorides, Phys. Rev. D 66 (2002)045017 arXiv:hep-th/0205272.

[11] A. I. Karanikas and C. N. Ktorides, Phys. Lett. B 560, 252 (2003) arXiv:hep-th/0301214.

[12] Z. Bern, L. Dixon and D. A. Kosower, JHEP01 (2000)027.

[13] A. S. Kapoyannis, A. I. Karanikas and C. N. Ktorides, arXiv:hep-th/0403148.

[14] A. Denner and S. Dittmaier, arXiv:hep-ph/0509141. 


\section{Tables}

$$
\left(\begin{array}{lll}
1 & 2 & 3 \\
1 & 2 & 4 \\
1 & 3 & 4 \\
2 & 3 & 4
\end{array}\right)
$$

Table 1. The output matrix of subroutine COMBINATIONASCEND with $L=4$ and

$$
K=3 .
$$

$$
\left(\begin{array}{ll}
1 & 0 \\
1 & 0 \\
2 & 0 \\
2 & 0 \\
3 & 0 \\
3 & 0
\end{array}\right) \rightarrow\left(\begin{array}{ll}
1 & 2 \\
1 & 3 \\
2 & 1 \\
2 & 3 \\
3 & 1 \\
3 & 2
\end{array}\right)
$$

Table 2. The gradual filling of the output matrix of subroutine ORDERS with $L=3$ and

$$
\begin{gathered}
K=2 . \\
\left(\begin{array}{llll}
1 & 3 & 2 & 4 \\
1 & 4 & 2 & 3 \\
1 & 2 & 3 & 4
\end{array}\right)
\end{gathered}
$$

Table 3. The output matrix of subroutine COMBINATIONPERTWO with $M=4$ and

$$
N=2 \text {. }
$$

\begin{tabular}{cr}
\hline Function & Representation \\
\hline$A_{n}$ & $200+n$ \\
$B_{n}$ & $300+n$ \\
$C_{n}$ & $n$ \\
$D_{n m}$ & $100 * n+m$ \\
\hline
\end{tabular}

Table 4. The representation of functions through integers in the output of subroutine MULTIPLYALL. The numbers $n, m$ are to be considered as two digit integers. 


\begin{tabular}{cc}
\hline Internal & Representation \\
Product & \\
\hline$p_{n} \cdot p_{m}$ & $200000+\mathrm{n}^{*} 100+200+\mathrm{m}$ \\
$\varepsilon^{n} \cdot p_{m}$ & $300000+\mathrm{n}^{*} 100+200+\mathrm{m}$ \\
$\varepsilon^{n} \cdot \varepsilon^{m}$ & $300000+\mathrm{n}^{*} 100+300+\mathrm{m}$ \\
\hline
\end{tabular}

Table 5. The representation of internal products through integers in the output of TRACE.

\begin{tabular}{cc}
\hline Function & Representation \\
\hline$\delta\left(u^{n+1}-u^{n}\right)$ & $300+\mathrm{n}$ \\
$\sum_{m \neq n} \varepsilon^{n} \cdot p_{m} \partial_{n} G\left(u_{n}, u_{m}\right)$ & $20000+\mathrm{n}^{*} 100+\mathrm{m}$ \\
$\varepsilon^{n} \cdot \varepsilon^{m} \partial_{n} \partial_{m} G\left(u_{n}, u_{m}\right)$ & $30000+\mathrm{n}^{*} 100+\mathrm{m}$ \\
& $\left(=30000+\mathrm{m}^{*} 100+\mathrm{n}\right)$ \\
\hline
\end{tabular}

Table 6. The representation of functions through integers in the output of TRACE.

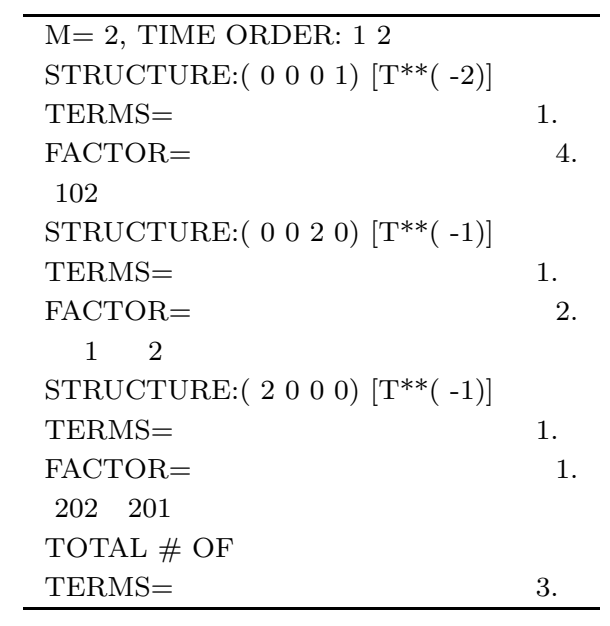

Table 7a. The output of MULTIPLYALL for $M=2$ and the time order $u_{1}<u_{2}$. 
$\mathrm{M}=2$, TIME ORDER: 12

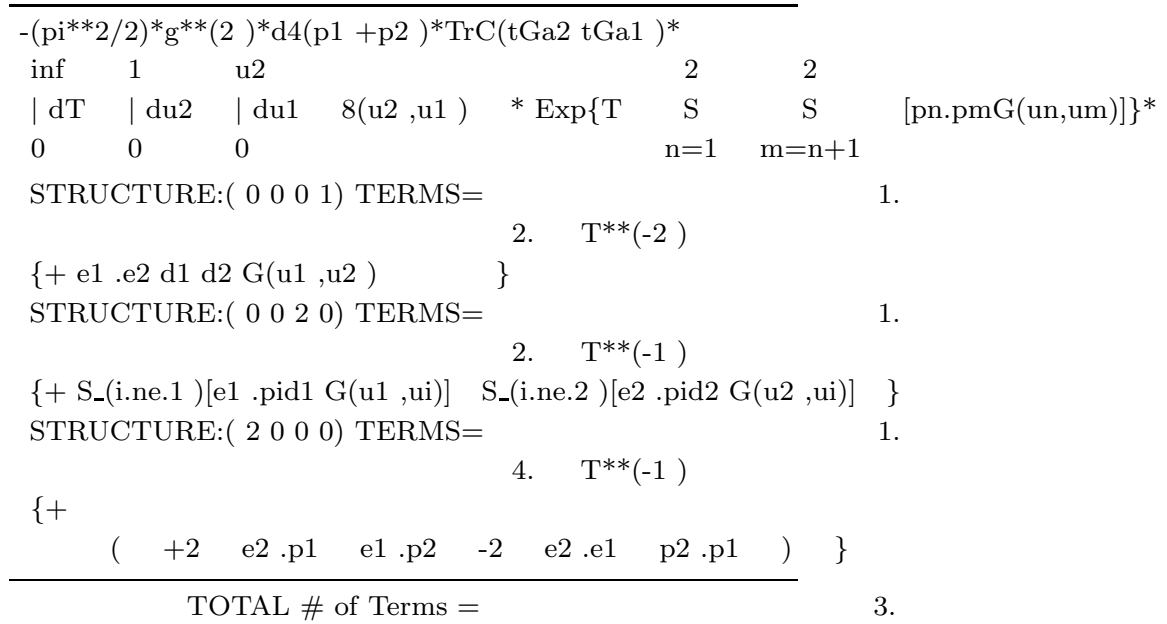

Table $7 b$. The function like output of TRACE for $M=2$ and the time order $u_{1}<u_{2}$. The Memorandum is similar to Table 8. 
$\mathrm{M}=3$, TIME ORDER: 312

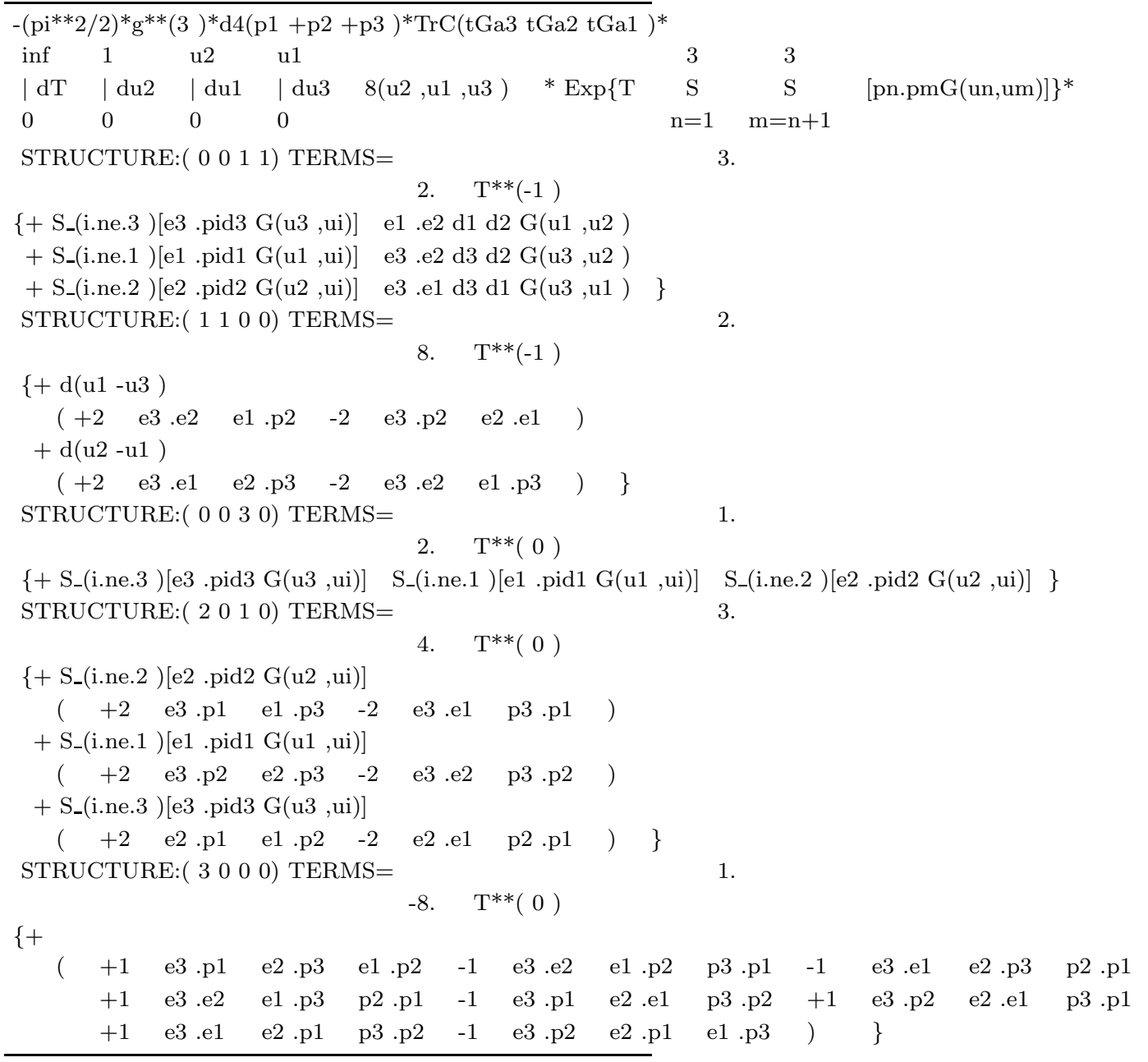

Table 8. The function like output of TRACE for $M=3$ and the time order $u_{3}<u_{1}<u_{2}$. 


\section{Figures}

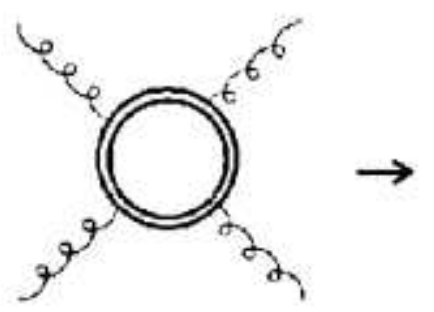

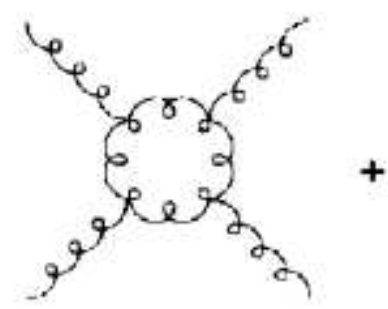

(a)

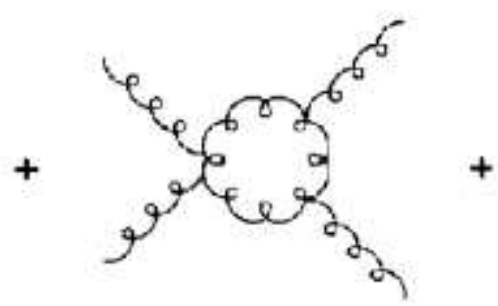

(b)

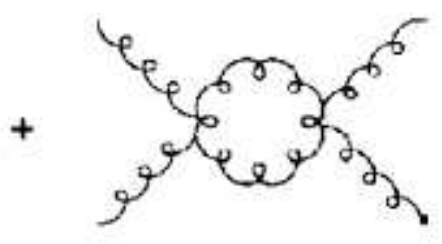

(c)

Figure 1 Illustration, for $M=4$, of the classes of one-gluon loop Feynman diagram (right side of arrow) which are simultaneously accommodated by the corresponding master formula depicted on left side of arrow. 


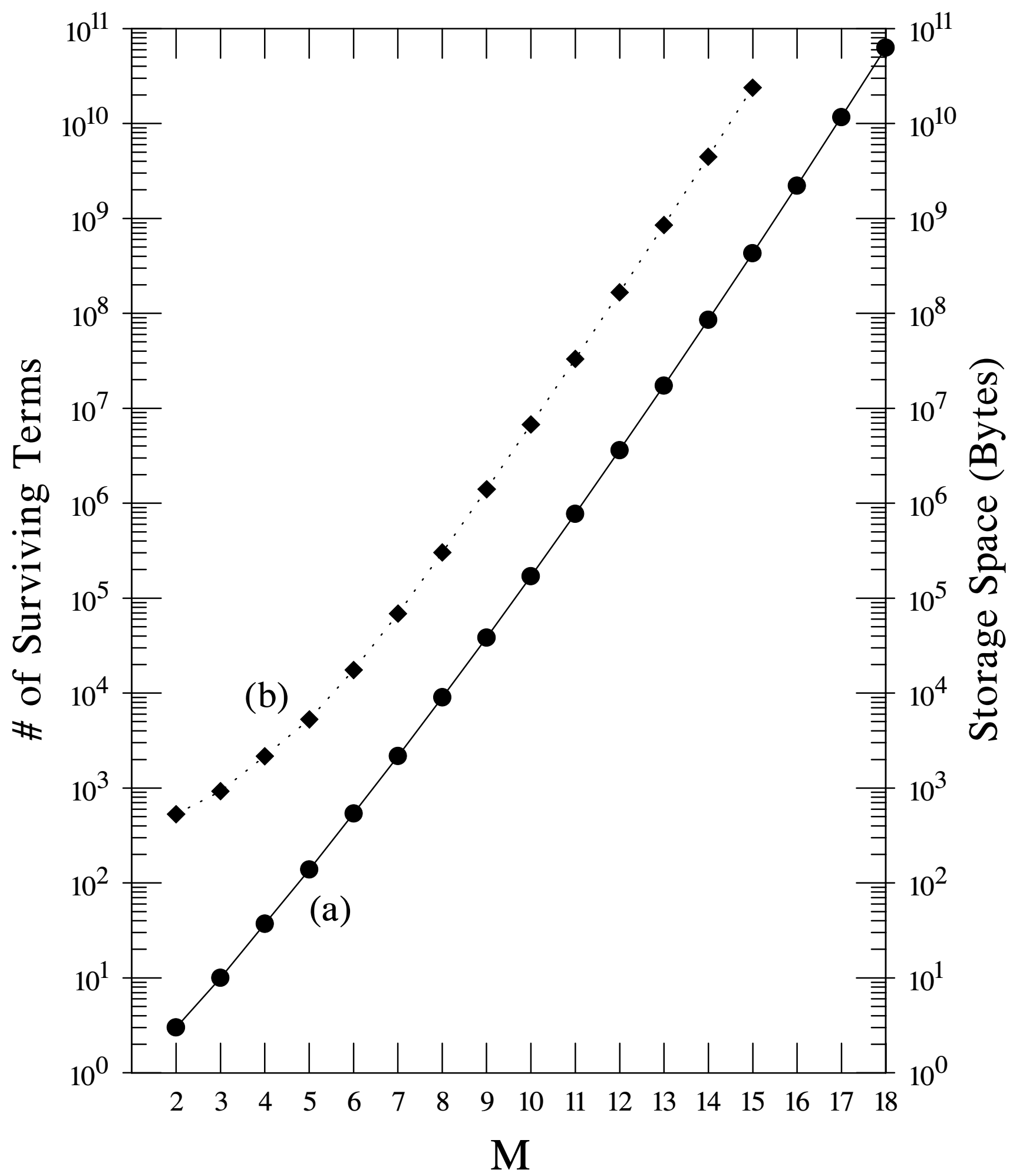

Figure 2 (a) The number of surviving terms (left axis) as function of $M$. (b) The storage space occupied by the output of subroutine MULTIPLYALL (right axis). 


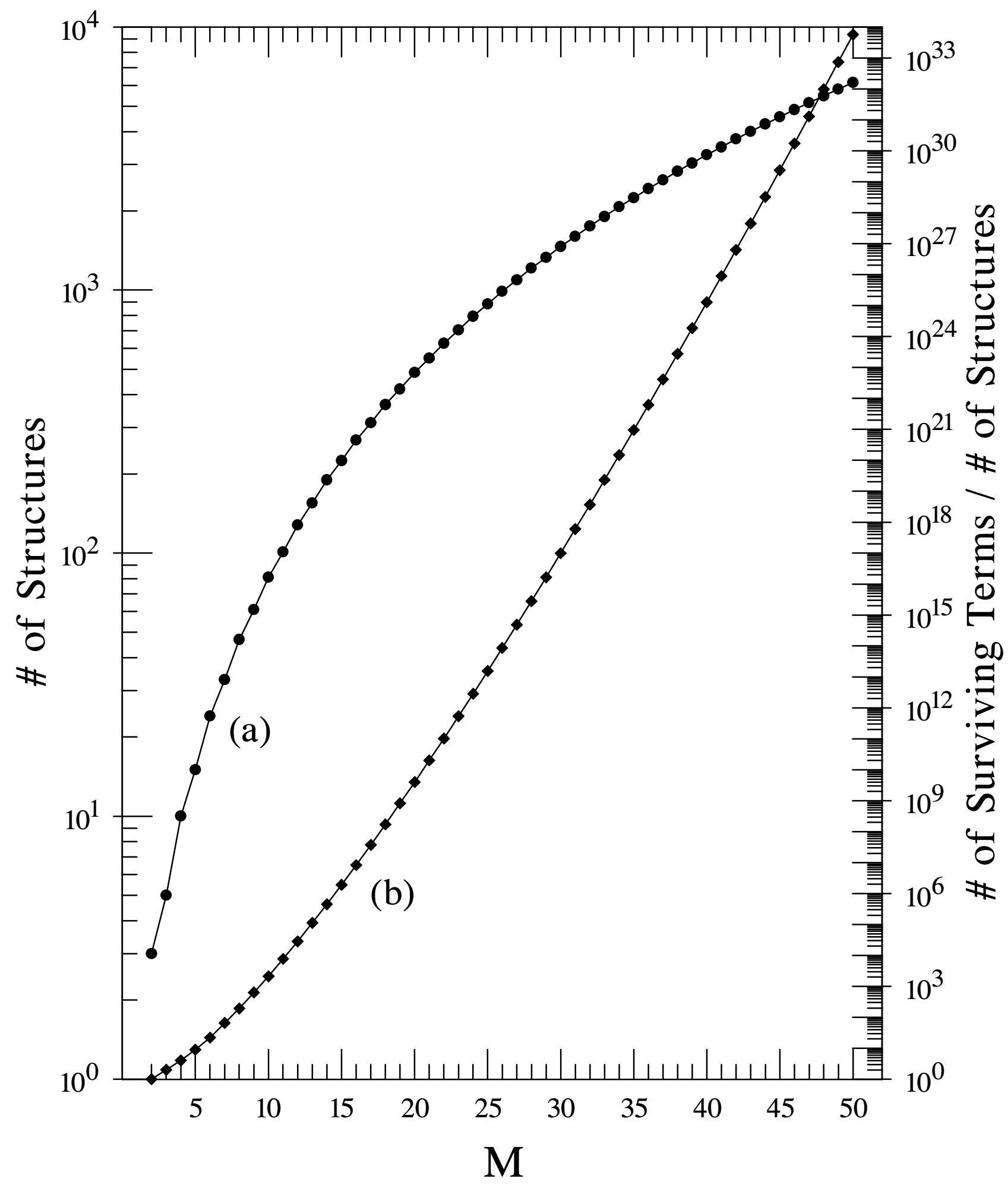

Figure 3 (a) The number of structures as function of $M$ (left axis). (b) The average number of surviving terms per structure as function of $M$ (right axis). 


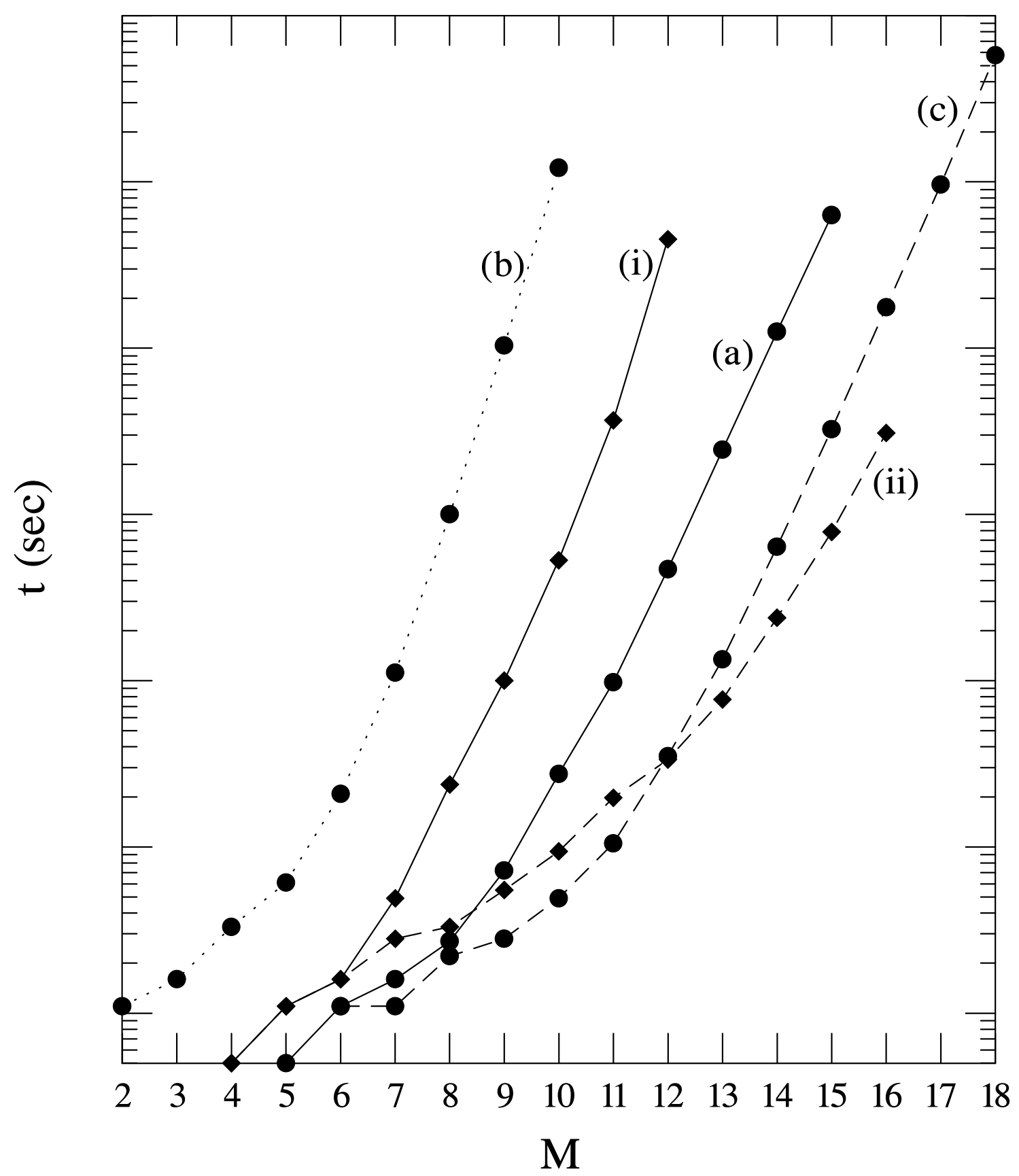

Figure 4 Curves (a)-(c) depict the consumed time for the completion of subroutine MULTIPLYALL as function of $M$. (a): Our new program with the output stored in files, (b): Our old program [13] with the output stored in files, (c): Our new program with suppressed output. Curves (i)-(ii) depict the consumed time for the completion of subroutine TRACE as function of $M$, for our new program. (i): Full result, (ii): Result with only the first term of each structure. (Time in all cases in measured using as processor INTEL CELERON at $1.8 \mathrm{GHz}$ ). 


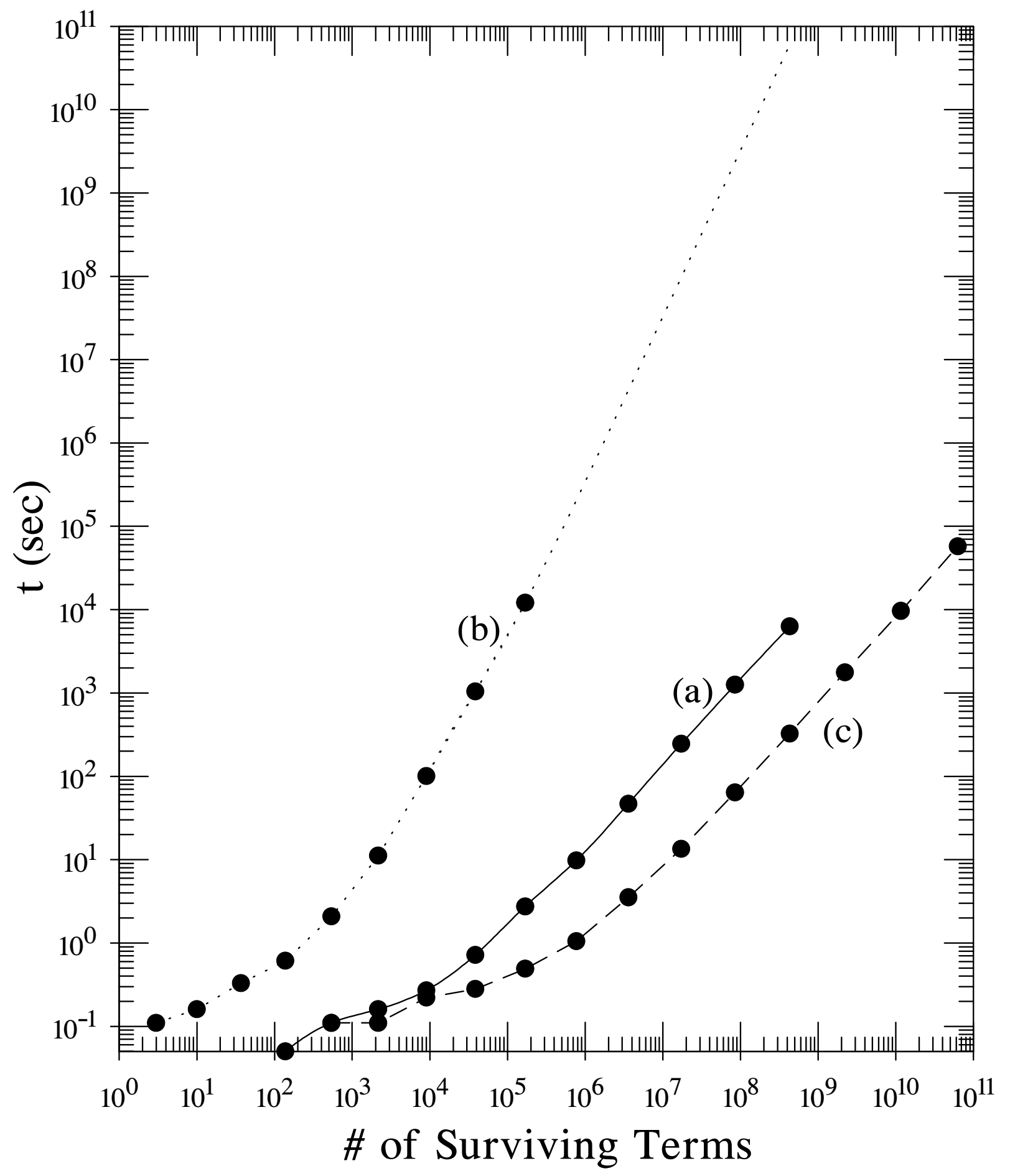

Figure 5 The consumed time in subroutine MULTIPLYALL ((a)-(c)) and TRACE ((i)-(ii)), as in Fig. 4, as function of the surviving terms in the final result. Curve (a) has been fitted with by a linear function and (b) has been extrapolated up to the surviving terms for $M=15$ by a quadratic function. 